\title{
CREATING THE CONDITIONS OF ANOMALOUS SELF-DIFFUSION IN A LIQUID WITH MOLECULAR DYNAMICS
}

\author{
J. Ryckebusch, S. Standaert, L. De Cruz \\ Department of Physics and Astronomy, Ghent University, Belgium
}

\begin{abstract}
We propose a computational method to simulate non-Gaussian self-diffusion in a simple liquid. The method is based on nonequilibrium molecular dynamics (NEMD).
\end{abstract}

Keywords: Nonequilibrium molecular dynamics, anomalous diffusion

PACS: 05.70.Ln, 05.10.-a

\section{INTRODUCTION}

A simple liquid is the prime example of a system of interacting units that possesses Gaussian self-diffusion properties. This implies that the mean square displacements are proportional with time $\Delta r^{2}(t) \sim t$. This is for example reflected in the Gaussian character of the single time step (defined by $\Delta t$ ) displacement distributions $P_{\Delta t}\left(\frac{\Delta x}{\sigma(t)}\right)$ with $\Delta x_{i}=$ $x_{i}(t+\Delta t)-x_{i}(t), i=1, \ldots, N$. The distribution $P_{\Delta t}\left(\frac{\Delta x}{\sigma(t)}\right)$ is one of the quantities that can be determined in a molecular-dynamics (MD) simulation of a system of $N$ molecules. Under equilibrium conditions, the width $\sigma$ of the distribution can be connected to the diffusion coefficient and depends on the temperature, on the intermolecular interaction, and on the density. We propose a simulation technique to create conditions of anomalous self-diffusion in a simple liquid. More in particular, we are seeking to simulate systems with a (non-Gaussian) fat-tailed $P_{\Delta t}\left(\frac{\Delta x}{\sigma(t)}\right)$. This implies that the $\Delta x$ 's are no longer confined to a certain scale (determined by the quantity $\sigma$ ) and that every so often molecules perform steps $\Delta x$ that are much larger than average.

\section{COMPUTATIONAL METHOD AND RESULTS}

Events of "all" sizes are encountered in systems which display self-organized criticality (SOC) and reside in a steady-state nonequilibrium state [1]. To summarize the major ideas of SOC: the system gets very slowly driven, which allows it to relax according to the rules of its internal dynamics. During the relaxation stage one typically observes events of all sizes.

We have translated the above-mentioned SOC ideas to a liquid as it is simulated in MD. We drive the simulation system by injecting potential energy at various locations in the simulation system at intermittent times. Clearly, this creates a temporal nonequilib- 
rium situation with a highly non-uniform potential energy density. Subsequently, we let the system evolve according to Newton's energy-conserving laws. The dissipation of the locally injected potential energy under conditions of constant total energy which characterizes the dynamics of the system, results in a small amount of very fast molecules. The anomalous feature of the self-diffusion properties of the system is reflected in nonGaussian single time step displacement distributions $P_{\Delta t}\left(\frac{\Delta x}{\sigma(t)}\right)$ during the nonequilibrium time periods of the simulation. One can discriminate between the equilibrium and nonequilibrium time periods of the simulation system by monitoring the average kinetic energy $\langle T\rangle$. During nonequilibrium time periods, the $\langle T\rangle$ is an increasing function of time.

In comparison to the equilibrium situation, we observe that under nonequilibrium conditions, small $\Delta x$ 's become more abundant, medium $\Delta x$ 's less probable, and that really large $\Delta x$ 's can be observed. In other words, the distribution $P_{\Delta t}\left(\frac{\Delta x}{\sigma(t)}\right)$ has fat tails, which is equivalent to saying that displacements $\Delta x$ of all sizes occur or that the self-diffusion properties are anomalous.

In the simulation we use an interaction potential $U_{S C}\left(r_{12}\right)$ with a soft-core repulsion at short inter-particles distances $r_{12}$ and an attractive part at medium $r_{12}$. Hard-core potentials are observed to drive the simulation system out of control under nonequilibrium conditions. The driving, or injection of potential energy, is achieved by subjecting the system to changes in the system's length scale at intermittent times. The rescaling modifies the intermolecular interaction according to $U_{S C}(r) \longrightarrow U_{S C}(\lambda(t) r)(\lambda(t)<1)$. The $\lambda$ gets modified in a stepwise fashion: $\lambda(t)=\lambda_{0}^{\left\lfloor\left(\frac{t}{\tau}\right)\right\rfloor}$, where $\left\lfloor\left(\frac{t}{\tau}\right)\right\rfloor$ rounds $\left(\frac{t}{\tau}\right)$ towards positive integer values. The parameter $\tau$ determines the time interval between two subsequent rescalings of $\lambda$. From the above it becomes clear that for a given temperature, density and interaction, the nonequilibrium features of the simulation are controlled by $\left(\lambda_{0}, \tau\right)$. The parameter $\tau$ is expressed in units of simulation steps. In line with typical SOC conditions [1], it is required that the driving is done in a sufficiently slow fashion. For a broad range of the two parameters $\lambda_{0}$ and $\tau$, we observe that our NEMD simulation technique generates conditions of anomalous self-diffusion. This proves the robustness of the proposed simulation method and hints at the fact that the anomalous self-diffusion is an emergent feature of the nonequilibrium simulation system. The "anomalous" character of $P_{\Delta t}\left(\frac{\Delta x}{\sigma(t)}\right)$ can be quantified by means of a non-vanishing fourth moment (or, kurtosis) of the distribution.

Summarizing, we have created a rather simple and robust simulation method to achieve conditions of anomalous self-diffusion in an interacting system. This emergence of global behaviour that cannot be determined from local properties is also a property of SOC, to which our simulation system bears similarities.

\section{REFERENCES}

1. Kim Christensen and Nicholas R. Moloney, Complexity and Criticality, Imperial College Press, London, 2005.

2. S. Standaert, J. Ryckebusch, L. De Cruz, Journal of Statistical Mechanics P04004 (2010). 